\title{
Crosstalk Between Type VI Secretion System and Mobile Genetic Elements
}

\author{
Arancha Peñil-Celis and M. Pilar Garcillán-Barcia* \\ Instituto de Biomedicina y Biotecnología de Cantabria, Universidad de Cantabria-Consejo Superior de Investigaciones \\ Científicas, Santander, Spain
}

Many bacterial processes require cell-cell contacts. Such are the cases of bacterial conjugation, one of the main horizontal gene transfer mechanisms that physically spreads DNA, and the type VI secretion systems (T6SSs), which deploy antibacterial activity. Bacteria depend on conjugation to adapt to changing environments, while T6SS killing activity could pose a threat to mating partners. Here we review the experimental evidences of overlapping and interaction between the T6SSs, bacterial conjugation, and conjugative genetic elements.

Keywords: type VI secretion system, bacterial conjugation, horizontal gene transfer, cell-cell communication, T6SS regulation, mobile genetic elements, plasmids, integrative and conjugative elements

\section{OPEN ACCESS INTRODUCTION}

Edited by:

Tatiana Venkova,

Fox Chase Cancer Center,

United States

Reviewed by:

Elisabeth Grohmann,

Beuth Hochschule für Technik

Berlin, Germany

Luis Rey,

Polytechnic University of

Madrid, Spain

*Correspondence:

M. Pilar Garcillán-Barcia

garcilmp@unican.es

Specialty section:

This article was submitted to

Molecular Recognition,

a section of the journal

Frontiers in Molecular Biosciences

Received: 23 September 2019 Accepted: 28 October 2019 Published: 13 November 2019

Citation:

Peñil-Celis A and Garcillán-Barcia MP (2019) Crosstalk Between Type VI

Secretion System and Mobile Genetic Elements. Front. Mol. Biosci. 6:126. doi: 10.3389/fmolb.2019.00126
Microbes often exist in complex multispecies communities where interaction is essential for keeping a balanced microbial ecosystem. Bacterial survival depends on the ability to succeed in the competition against rival bacterial cells. Critical to this goal is the type VI secretion system (T6SS), a macromolecular multiprotein complex dedicated to the delivery of toxins across the cell envelope of the donor bacteria (predator) into the cytoplasm of a target cell (prey), either another bacterium, or an eukaryotic cell [for recent reviews on T6SS structure, assembly, and activity, see (Cianfanelli et al., 2016; Nazarov et al., 2018; Nguyen et al., 2018; Cherrak et al., 2019; Wang et al., 2019). Only a few phyla of Gram-negative microorganisms encode T6SSs (Abby et al., 2016), suggesting a role for horizontal gene transfer (HGT) in its origin. Four T6SS phylogenetic subtypes are distinguished (Russell et al., 2014; Böck et al., 2017). Several protein complexes compose the T6SS most common subtype (T6SS ${ }^{\text {i }}$ : a membrane complex (Durand et al., 2012, 2015; Rapisarda et al., 2019), which encompasses proteins Tss], TssL, and TssM, a baseplate [TssE, TssF, TssG, and TssK (Nazarov et al., 2018; Park et al., 2018; Liebl et al., 2019)], a syringe (Wang et al., 2017), which is in turn composed of an inner tube that is secreted by the functional system [Hcp (Douzi et al., 2014)], a needle spike [VgrG (Uchida et al., 2014) and PAAR (Shneider et al., 2013)], and a sheath [TssB and TssC (Kudryashev et al., 2015)]. Furthermore, a sheath assembly protein, TssA (Schneider et al., 2019), and a sheath disassembly ATPase, TssH (also known as ClpV) (Pietrosiuk et al., 2011) are part of the system. A plethora of T6SS protein effectors and their corresponding immunity proteins have been identified (Durand et al., 2014; Kostiuk et al., 2017; Lien and Lai, 2017).

A claimed common evolutionary origin for T6SSs and contractile tailed-bacteriophages is supported by the conservation of some of their components (Hcp, VgrG, TssC, TssF, TssA, TssK, TssG, and TssE (Pukatzki et al., 2007; Leiman et al., 2009; Lossi et al., 2011; Planamente et al., 2016), and the similarities in the assembly mechanisms between the T6SS and the bacteriophage tail tube-sheath (Veesler and Cambillau, 2011; Lossi et al., 2013; Brunet et al., 2014). Accretion of DotU/IcmH and IcmF homologs of the Dot/Icm T4SS of Legionella pneumophila (Christie, 2016), TssL and TssM, which mediate the polar targeting of such T4SS (Ghosal et al., 2019), provides a docking station for the phage-like T66S device (Durand et al., 2015). 
Most T6SSs are functional in a cell-cell contact-dependent way. Cell-cell communication mechanisms are thus expected to have an impact on T6SS activity. Several examples support the T6SS regulation by quorum sensing (QS) networks (Pena et al., 2019). A phosphorylation cascade that mediates a positive regulatory loop entwining T6SS and QS was found in Vibrio alginolyticus (Yang et al., 2018). In Vibrio cholerae, expression of Hcp showed to be growth phase-dependent, positively and negatively regulated by the QS regulators HapR and LuxO, respectively (Ishikawa et al., 2009; Zheng et al., 2010). Biofilm formation and T6SS expression in Burkolderia cenocepacia were upregulated by QS (Aubert et al., 2013), while expression of T6SS effector and immunity genes of Burkholderia thailandensis was also activated by QS (Majerczyk et al., 2016). In the case of Pseudomonas aeruginosa, its three T6SSs were differentially regulated by QS: whereas QS regulators LasR and MvfR suppressed the expression of H1-T6SS, they positively regulated that of H2- and H3-T6SS (Lesic et al., 2009). On the other hand, although cell-cell contact is not a requirement for natural transformation, in Vibrio cholerae, T6SS is part of the competence regulon and functions as a transformation enhancer for DNA acquisition (Borgeaud et al., 2015; Veening and Blokesch, 2017; Matthey et al., 2019). Competence-mediated induction of T6SS released DNA and made it accessible for HGT in Vibrio cholerae. Besides, competence-induced T6SS-mediated killing increased the natural transformation efficiency and boosted the acquisition of large genomic regions from killed neighbors (Borgeaud et al., 2015), including novel functional T6SS effector-immunity pairs (Thomas et al., 2017).

The above results suggest that T6SS and cell-cell communication processes are interwoven. Here we will focus on the contribution of another intercellular mechanism, bacterial conjugation, and its associated players, plasmids and integrative and conjugative elements (ICEs), to the activity of T6SS.

\section{T6SS CONTROL BY PLASMID-ENCODED REGULATORS}

T6SSs are implicated in a wide range of functions and regulated by a large diversity of mechanisms (Bernard et al., 2010; Alteri and Mobley, 2016). Transcriptional regulators of T6SS loci have been detected in plasmids. The first report on a T6SS regulation by a plasmid-encoded regulator was that of Sci-2 (Figure 1A). Sci-2 T6SS is encoded in entero-aggregative, avian-pathogenic and Shiga toxin-producing Escherichia coli strains (Journet and Cascales, 2016). It confers a growth advantage to enteroaggregative E. coli (EAEC) by causing non-immune E. coli killing (Brunet et al., 2013). The prototype EAEC strain 042 carries the pAA2 plasmid, which encodes a transcriptional regulator of the AraC family, the $\operatorname{aggR}$ gene (Nataro et al., 1994). AggR plays a central role in modulating adherence of EAEC 042 by activating plasmid-borne genes, such as the attachment adherence fimbriae aafDA (Elias et al., 1999), the anti-aggregative protein dispersin aap (Sheikh et al., 2002), and the T1SS aatPABCD for Aap transport (Nishi et al., 2003). EAEC chromosomal genes located in the pathogenicity island PAI-1 are also included in the AggR regulon (Dudley et al., 2006; Morin et al., 2013; Yasir et al., 2019). Among these genes, a cluster designated aaiA-P (Sci-2) was found expressed at least 2 -fold higher in the wild-type strain 042 than in the aggR- derivative using a microarray approach (Dudley et al., 2006). Two proteins of this cluster were identified by mass spectrometry analysis in the whole-cell proteome of the wild-type strain, while they were absent in the aggR-derivative. One of
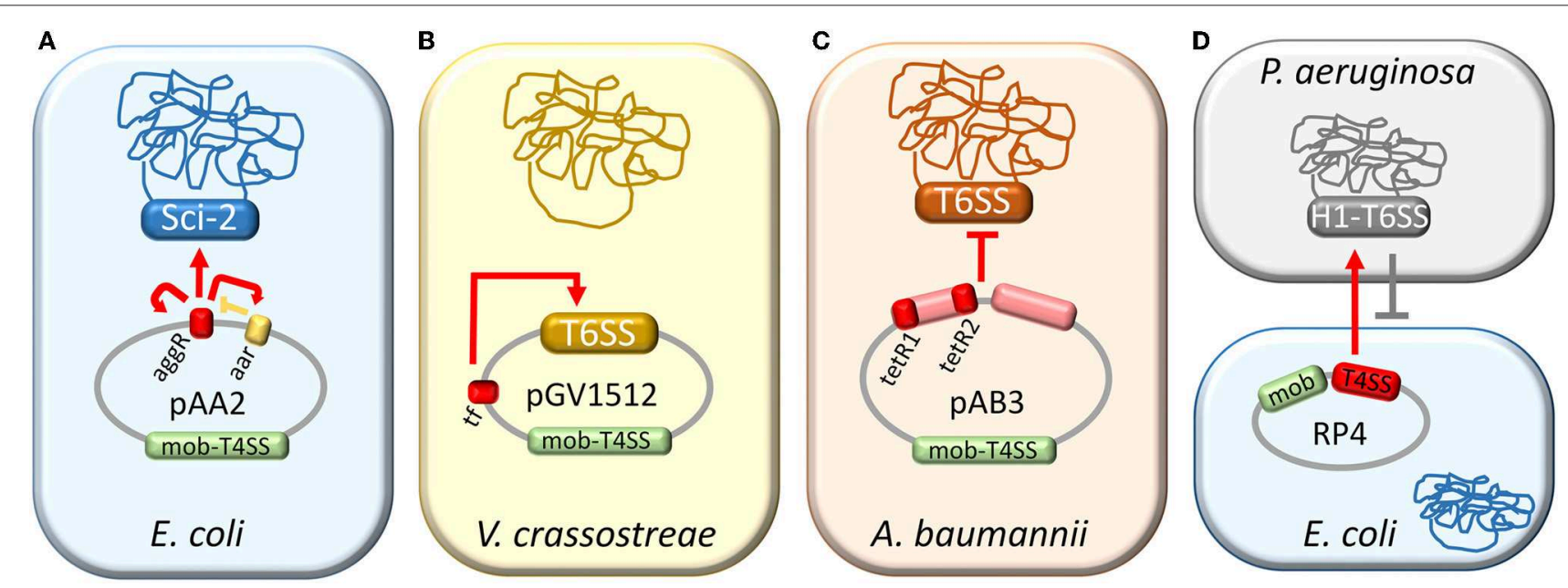

FIGURE 1 | Control of T6SS activity by plasmids. Plasmid-mediated regulation of T6SSs in E. coli (A), V. crassostreae (B), A. baumannii (C), and P. aeruginosa (D). Plasmid regions involved in T6SS regulation are depicted in red. (A) pAA2-encoded auto-regulator AggR activates the Sci-2 T6SS (red arrows) and is negatively regulated by Aar (yellow lines). (B) Transcriptional factor (TF) of pGV1512 activates the plasmid-encoded T6SS (red arrow). (C) Two regions of the pAB3 plasmid, one of them including tetR-like regulators, repress the T6SS of $A$. baumannii (red lines) (D) Cell-cell interactions mediated by the T4SS of plasmid RP4 trigger H1-T6SS counterattack (red arrow), resulting in a decreased survival specifically in plasmid-bearing cells (gray lines). 
these proteins, AaiC ( $\mathrm{Hcp}$ ) was also detected in the supernatant of exponentially-growing cells cultured in DMEM (Dudley et al., 2006), a condition that induces AggR production (Sheikh et al., 2001). An AggR-dependent promoter was localized in the region comprised from $466 \mathrm{bp}$ upstream to $300 \mathrm{bp}$ downstream of the predicted aaiA translational start site (Dudley et al., 2006). In the wild-type strain, the expression from this promoter was approximately 3 -fold over that seen in the aggR mutant.

AggR was found to autoactivate its expression (Morin et al., 2010). By primer extension analysis, the transcriptional start site of the $\operatorname{aggR}$ promoter (PaggR) was located 40 nucleotides upstream of the translational start (Morin et al., 2010). DNA footprinting experiments revealed the presence of two AggR-binding sites: one upstream of the transcriptional start site and one downstream. A consensus sequence resembling sites for the Rns regulator from enterotoxigenic E. coli (Munson, 2013) was found for AggR binding: ANNNNNNTATC (Morin et al., 2010). EAEC plasmid and chromosomal AggRregulated genes identified by RNAseq were found to contain a WWWWWWWTATC (where W means A or T) sequence spaced 21-23 bp upstream of the -10 promoter elements (Yasir et al., 2019). AggR is negatively controlled by the plasmid-encoded Aar repressor protein through direct binding (Santiago et al., 2016), while the aar gene is in turn positively regulated by AggR (Santiago et al., 2014). It remains unknown what environmental signal is sensed by AggR (Morin et al., 2013).

Another example of T6SS regulation by an AraC-like activator is provided by pGV1512, a conjugative plasmid that invaded Vibrio crassostreae, turning it from a benign oyster commensal into a pathogen (Bruto et al., 2017; Figure 1B). This plasmid encodes a T6SS organized into two divergently-transcribed operons. A plasmid region located between T6SS and T4SS, Px3, was found to be necessary for $V$. crassostreae virulence (Bruto et al., 2017). Px3 encodes a transcriptional regulator (TF) of the AraC family, which activated the transcription of plasmidencoded T6SS genes, as shown by RNAseq analysis, and restored the virulence to a $\Delta$ Px3 mutant (Piel et al., 2019). Two functional promoters were located in the intergenic region between T6SS operons. Deletion of a palindromic sequence of six nucleotides spaced by five nucleotides, located between both promoters, altered the induction capacity of TF (Piel et al., 2019).

Negative transcriptional regulation exerted by a plasmid to a chromosomally-encoded T6SS has been also documented in Acinetobacter baumannii (Figure 1C). T6SSs are conserved and syntenic among A. baumannii strains (Weber et al., 2013). A. baumannii ATCC 17978 constitutively produces and secretes Hcp, a hallmark of an active T6SS, but initial efforts to observe a predator phenotype failed. There were no significant differences in the survival of any of the preys (E. coli, A. baumannii, A. nosocomialis, A. baylyi) in different experimental conditions tested (Weber et al., 2013). Nevertheless, when individual colonies from a clinical isolate of A. baumannii (strain Ab 04) were analyzed for Hcp secretion by ELISA, two contrasting profiles were found (Weber et al., 2015). Some colonies behaved coherently with a T6SS- and others with a T6SS+ phenotype. Whole-genome sequencing of both Ab 04 subpopulations revealed that their genomes encoded a T6SS, though they differed in the carriage of a multidrug-resistance conjugative plasmid (pAB04-1), absent in the cells exhibiting the T6SS+ phenotype.

This plasmid was not fully stable in A. baumannii, so a subpopulation lost it, rendering colonies that produced and secreted Hcp while becoming susceptible to antimicrobials. Colonies displaying the \pm T6SS phenotypes were also isolated for A. baumannii strains 17978 and 1438 (Weber et al., 2015). In all cases, T6SS+ cells efficiently killed $E$. coli in competition assays, in contrast to their T6SS- counterparts. This killing was dependent on a functional T6SS, as verified by using mutants of essential T6SS components, which did not kill E. coli. Mating experiments in which the transmissible plasmid was transferred from donor A. baumannii cells with the T6SS- phenotype to T6SS+ recipient cells rendered transconjugants deficient in bacterial killing. These experiments provided evidence that pAB04-1 encoded the genetic determinants for silencing the $A$. baumannii T6SS activity.

The pAB04-1 backbone is commonly found in other $A$. baumannii strains, and the plasmid variants mainly differ in their antimicrobial-resistance cargoes (Weber et al., 2015). A. baumannii prototype strain, ATCC 17978, harbors a pAB04-1-like plasmid, pAB3. Transferred to either a clonallyunrelated A. baumannii strain, A. baylyi or A. nosocomialis, the transconjugants did not secrete Hcp, indicating that the T6SSs of these strains were also susceptible to repression by the plasmid and thus pointing to a common repression mechanism in a broad range of Acinetobacter species (Di Venanzio et al., 2019b). Another pAB04-1-like plasmid, pAB5, from A. baumannii strain UPAB1, was shown to regulate the expression of multiple chromosomally-encoded virulence factors, including T6SS (Di Venanzio et al., 2019a). An UPAB1 derivative lacking pAB5 displayed increased susceptibility to multiple antibiotics and activation of the T6SS, as observed by secretion of the T6SSassociated protein Hcp. Two tetR-like regulators were found to be encoded only in pAB04-1-like plasmids (Weber et al., 2015). The deletion of either $\mathrm{pAB} 3$ tetR gene or both increased Hcp expression but did not restore Hcp secretion (Di Venanzio et al., 2019b). The deletion of the tetR-like repressor genes was thus not enough to trigger per se the T6SS activity. Nevertheless, when these regulators were overexpressed in a T6SS+ phenotype ATCC 17978 population, Hcp expression and secretion drastically decreased, and this transformed population was impaired as a predator in its E. coli killing ability (Weber et al., 2015).

\section{T6SS ACTIVITY AFFECTS BACTERIAL CONJUGATION}

P. aeruginosa senses exogenous attacks by the T6SS of akin and non-akin bacteria and post-translationally activates its H1T6SS at the precise location of the initial strikes (Basler and Mekalanos, 2012; Basler et al., 2013). Other perturbations of the cell envelope, such as the presence of the Gram-negative bacterial 
membrane disruptor polymyxin B, also increased the H1-T6SS activity (Ho et al., 2013). These results suggested that membrane perturbations trigger the H1-T6SS activity.

Bacterial conjugation is an HGT mechanism that involves contact between the cell envelopes of the mating-pair partners, i.e., donor and recipient cells. It is essentially mediated by plasmids and integrative and conjugative elements (ICEs) (Smillie et al., 2010; Guglielmini et al., 2011). These genomic platforms encode the genetic requirements for their transfer: a mob region containing an origin of transfer (the single element strictly required in cis), a relaxase (Smillie et al., 2010; Zechner et al., 2017; Guzmán-Herrador and Llosa, 2019), and a type IV coupling protein (Gomis-Rüth et al., 2004; Peña and Arechaga, 2013); and a mating-pair bridge composed of a type IV secretion system (T4SS) (Cabezón et al., 2015; Bergé et al., 2017; Grohmann et al., 2018; Li et al., 2019).

Donor E. coli strains bearing broad host-range conjugative plasmids RP4/RK2 or pKM101, but not the narrow hostrange plasmid $\mathrm{F}$, were more sensitive to killing by a T6SS+ $P$. aeruginosa recipient than plasmid-lacking donors (Ho et al., 2013; Figure 1D). Furthermore, RP4-containing E. coli donors were selectively killed by $P$. aeruginosa in a mixed culture with RP4-lacking E. coli cells. P. aeruginosa mutants defective in the attack-sensing pathway genes tagT and $p p p A$ exhibited greater conjugation efficiency as recipient strains and did not kill RP4bearing E. coli donors. Transposon mutagenesis of RP4 rendered T4SS mutants with impaired ability to transfer and induce T6SS donor-directed killing response in $P$. aeruginosa. RP4 mutants in the relaxosome components genes traI and traJ and the coupling protein gene traG were also defective in DNA conjugation but showed increased donor-directed T6SS response compared to wild type RP4, suggesting that successful DNA transfer was not necessary to activate a T6SS attack by $P$. aeruginosa. So, mating donors can trigger a T6SS response in the recipient, which in turn causes a decrease in their survival.

In the case of $A$. baumannii, conjugative dissemination of the pAB4-01/pAB3 plasmids relies on the repression of the T6SS encoded in the donor strain. To evaluate the impact of the plasmid-mediated T6SS repression in plasmid dissemination through conjugation, $\mathrm{pAB} 3$ derivatives were used in mating assays (Di Venanzio et al., 2019b). One of them, pAB3 $\Delta$ tetR1,2 lacked genes ACX60_RS18875-ACX60_RS18900 (GenBank Acc. No. NZ_CP012005.1), a region that contains the tetR-like genes tetR1 and tetR2. On this mutant, a second deletion comprising genes ACX60_RS18760-ACX60_RS18795 was introduced, producing plasmid $\mathrm{pAB}^{*}$. Wild-type and mutant plasmids were efficiently transferred between isogenic A. baumannii ATCC 17,978 strains (T6SS-resistant). When a non-immunogenic and thus T6SS-susceptible ATCC 17978 derivative was used as a recipient, $\mathrm{pAB} 3$ and $\mathrm{pAB} 3 \Delta \mathrm{tet} R 1,2$ transfer efficiencies were not altered. Nevertheless, the transfer of the mutant plasmid $\mathrm{pAB}^{*}$ and that of mobilizable plasmids relying on $\mathrm{pAB}^{*}$ as a helper were practically abolished due to recipient killing mediated by the donor's T6SS. Congruently, in competition experiments pAB3 overcame the mutant plasmid pAB3* at invading a T6SS-susceptible population. On the other hand, the efficiency of plasmid conjugation was also deeply affected when the recipient strain was T6SS-proficient and nonisogenic to the donor. pAB3-like plasmids would thus seem to guarantee their transmission by preventing T6SS-mediated killing of non-isogenic recipient strains, depending on their capacity to repress T6SS activity in the donor cells.

\section{T6SSs ENCODED IN MOBILE PLATFORMS}

The above results point to T6SS and conjugation as incompatible processes. Plasmids and ICEs are mobile genetic platforms that rely on bacterial conjugation for their dissemination, and thus do not seem a priori good platforms for T6SS. Plasmids have a predominant role as genetic couriers (Halary et al., 2010). Twenty-nine plasmids encoding T6SS genes are listed in the T6SS database SecReT6 (http://db-mml.sjtu.edu.cn/SecReT6/) (Li et al., 2015). Experimental evidence on these T6SSs is available only for that encoded in the $2.1 \mathrm{Mb}$ megaplasmid pGMI1000MP of the plant pathogen Ralstonia solanacearum GMI1000. A strain containing a tssB mutant of this plasmid was reported to be impaired in Hcp secretion and biofilm formation (Zhang et al., 2014). It had also significantly attenuated its virulence on tomato plants. Megaplasmids from other $R$. solanacearum strains also encode T6SS genes: RCFBPv3_mp, CMR15_mp, FQY_4 megaplasmid, Po82 megaplasmid, and mpPSI07. Plasmids pESA3 (Cronobacter sakazakii) and pEA320 (Pantoea ananatis) are also recorded in SecReT6. The existence of plasmids encoding T6SS genes in these species does not seem anecdotal. Using PCR probes based on the T6SS of pESA3, at least a partial T6SS cluster was detected in 175 out of 177 plasmid-harboring C. sakazakii strains analyzed (Franco et al., 2011) and comparative genomics of T6SSs in strains of $P$. ananatis from different environments revealed a plasmid-borne T6SS in a third of the analyzed strains (Shyntum et al., 2014). This T6SS type was restricted to strains of $P$. ananatis isolated from symptomatic plant material, suggesting the possibility of an association between the plasmid-borne T6SS and either pathogenicity or host specificity.

Out of the SecReT6 database, and besides the abovementioned in pGV1512, a functional T6SS was reported in the Rizobium etli Mim1 megaplasmid pRETMiM1f (SalineroLanzarote et al., 2019). Immunodetection of Hcp protein indicated that this T6SS was active at high cell densities, in the presence of root exudates, and in bean nodules. $R$. etli T6SSmutants produced plants with lower dry weight and smaller nodules than the wild-type strain, indicating for the first time that the T6SS played a positive role in Rhizobium-legume symbiosis.

ICEs are the most abundant conjugative elements in practically all prokaryotic clades (Guglielmini et al., 2011). Genome analysis strongly suggested transfer of the T6SS $S^{\text {iii }}$ subtype mediated by an ICE between Bacteroidales strains within the human gut ecosystem (Coyne et al., 2014). T6SS ${ }^{\mathrm{iii}}$ has been exclusively found in Bacteroidetes (Abby et al., 2016) and functions in a mechanistically similar manner to $\mathrm{T}^{2} \mathrm{SS}^{\mathrm{i}}$ to target competitor bacteria (Russell et al., 2014). T6SS ${ }^{\text {iii }}$ loci were found in more than half of human gut Bacteroidales strains, and they segregated into three evolutionarily-distinct genetic 
architectures, two of which were located on ICEs, and one of them had been transferred among co-resident Bacteroidales species in the human gut (Coyne et al., 2016). Low diversity in the effector-immunity pairs of the ICE-encoded T6SS ${ }^{\mathrm{iii}}$ was detected in human microbiome samples, an indicator of neighbor compatibility likely facilitated by HGT through ICE conjugation (Verster et al., 2017).

\section{CONCLUSIONS}

The antibacterial activity of T6SS affects bacterial conjugation by either killing plasmid-bearing donors or non-immune recipients and thus could undermine the DNA spreading. In turn, conjugative platforms (plasmids and ICEs) encode regulatory elements for controlling the T6SS activity. More intriguing is the fact that T6SS is not confined to the chromosome, but also

\section{REFERENCES}

Abby, S. S., Cury, J., Guglielmini, J., Néron, B., Touchon, M., and Rocha, E. P. C. (2016). Identification of protein secretion systems in bacterial genomes. Sci. Rep. 6:23080. doi: 10.1038/srep23080

Alteri, C. J., and Mobley, H. L. T. (2016). The versatile type VI secretion system. Microbiol. Spectr. 4, 1-26. doi: 10.1128/microbiolspec.VMBF-0026-2015

Aubert, D. F., O'Grady, E. P., Hamad, M. A., Sokol, P. A., and Valvano, M. A. (2013). The Burkholderia cenocepacia sensor kinase hybrid AtsR is a global regulator modulating quorum-sensing signalling. Environ. Microbiol. 15, 372-385. doi: 10.1111/j.1462-2920.2012.02828.x

Basler, M., Ho, B. T., and Mekalanos, J. J. (2013). Tit-for-tat: type VI secretion system counterattack during bacterial cell-cell interactions. Cell 152, 884-894. doi: 10.1016/j.cell.2013.01.042

Basler, M., and Mekalanos, J. J. (2012). Type 6 secretion dynamics within and between bacterial cells. Science 337:815. doi: 10.1126/science.1222901

Bergé, C., Waksman, G., and Terradot, L. (2017). Structural and molecular biology of type IV secretion systems. Curr. Top. Microbiol. Immunol. 413, 31-60. doi: 10.1007/978-3-319-75241-9_2

Bernard, C. S., Brunet, Y. R., Gueguen, E., and Cascales, E. (2010). Nooks and crannies in type VI secretion regulation. J. Bacteriol. 192, 3850-3860. doi: 10.1128/JB.00370-10

Böck, D., Medeiros, J. M., Tsao, H.-F., Penz, T., Weiss, G. L., Aistleitner, K., et al. (2017). In situ architecture, function, and evolution of a contractile injection system. Science 357, 713-717. doi: 10.1126/science.aan7904

Borgeaud, S., Metzger, L. C., Scrignari, T., and Blokesch, M. (2015). The type VI secretion system of Vibrio cholerae fosters horizontal gene transfer. Science 347, 63-67. doi: 10.1126/science.1260064

Brunet, Y. R., Espinosa, L., Harchouni, S., Mignot, T., and Cascales, E. (2013). Imaging type VI secretion-mediated bacterial killing. Cell Rep. 3, 36-41. doi: $10.1016 /$ j.celrep.2012.11.027

Brunet, Y. R., Hénin, J., Celia, H., and Cascales, E. (2014). Type VI secretion and bacteriophage tail tubes share a common assembly pathway. EMBO Rep. 15, 315-321. doi: 10.1002/embr.201337936

Bruto, M., James, A., Petton, B., Labreuche, Y., Chenivesse, S., AlunnoBruscia, M., et al. (2017). Vibrio crassostreae, a benign oyster colonizer turned into a pathogen after plasmid acquisition. ISME J. 11, 1043-1052. doi: 10.1038/ismej.2016.162

Cabezón, E., Ripoll-Rozada, J., Peña, A., de la Cruz, F., and Arechaga, I. (2015). Towards an integrated model of bacterial conjugation. FEMS Microbiol. Rev. 39, 81-95. doi: 10.1111/1574-6976.12085

Cherrak, Y., Flaugnatti, N., Durand, E., Journet, L., and Cascales, E. (2019). Structure and activity of the type VI secretion system. Microbiol. Spectr. 7, 1-11. doi: 10.1128/microbiolspec.PSIB-0031-2019

Christie, P. J. (2016). The mosaic type IV secretion systems. EcoSal Plus 7, 1-34. doi: 10.1128/ecosalplus.ESP-0020-2015 present in these mobile platforms. How plasmids and ICEs have found their ways to deal with this weaponry carriage without sacrificing their transfer potential remains to be researched.

\section{AUTHOR CONTRIBUTIONS}

MG-B conceived the study. AP-C and MG-B wrote the manuscript.

\section{FUNDING}

This work was supported by Consejo Superior de Investigaciones Científicas (201820I143 to MG-B). We acknowledge support of the publication fee by the CSIC Open Access Publication Support Initiative through its Unit of Information Resources for Research (URICI).
Cianfanelli, F. R., Monlezun, L., and Coulthurst, S. J. (2016). Aim, load, fire: the type VI secretion system, a bacterial nanoweapon. Trends Microbiol. 24, 51-62. doi: 10.1016/j.tim.2015.10.005

Coyne, M. J., Roelofs, K. G., and Comstock, L. E. (2016). Type VI secretion systems of human gut bacteroidales segregate into three genetic architectures, two of which are contained on mobile genetic elements. BMC Genomics 17:58. doi: 10.1186/s12864-016-2377-z

Coyne, M. J., Zitomersky, N. L., McGuire, A. M., Earl, A. M., and Comstock, L. E. (2014). Evidence of extensive DNA transfer between bacteroidales species within the human gut. MBio 5, e01305-e01314. doi: 10.1128/mBio.01 305-14

Di Venanzio, G., Flores-Mireles, A. L., Calix, J. J., Haurat, M. F., Scott, N. E., Palmer, L. D., et al. (2019a). Urinary tract colonization is enhanced by a plasmid that regulates uropathogenic Acinetobacter baumannii chromosomal genes. Nat. Commun. 10:2763. doi: 10.1038/s41467-01910706-y

Di Venanzio, G., Moon, K. H., Weber, B. S., Lopez, J., Ly, P. M., Potter, R. F., et al. (2019b). Multidrug-resistant plasmids repress chromosomally encoded T6SS to enable their dissemination. Proc. Natl. Acad. Sci. U.S.A. 116, 1378-1383. doi: 10.1073/pnas.1812557116

Douzi, B., Spinelli, S., Blangy, S., Roussel, A., Durand, E., Brunet, Y. R., et al. (2014). Crystal structure and self-interaction of the type VI secretion tailtube protein from enteroaggregative Escherichia coli. PLOS ONE 9:e86918. doi: 10.1371/journal.pone.0086918

Dudley, E. G., Thomson, N. R., Parkhill, J., Morin, N. P., and Nataro, J. P. (2006). Proteomic and microarray characterization of the AggR regulon identifies a pheU pathogenicity island in enteroaggregative Escherichia coli. Mol. Microbiol. 61, 1267-1282. doi: 10.1111/j.1365-2958.2006.05281.x

Durand, E., Cambillau, C., Cascales, E., and Journet, L. (2014). VgrG, Tae, Tle, and beyond: the versatile arsenal of Type VI secretion effectors. Trends Microbiol. 22, 498-507. doi: 10.1016/j.tim.2014.06.004

Durand, E., Nguyen, V. S., Zoued, A., Logger, L., Spinelli, S., Desmyter, A., et al. (2015). Biogenesis and structure of a type VI secretion membrane core complex. Nature 523, 555-560. doi: 10.1038/nature14667

Durand, E., Zoued, A., Spinelli, S., Watson, P. J. H., Aschtgen, M.-S., Journet, L., et al. (2012). Structural characterization and oligomerization of the TssL protein, a component shared by bacterial type VI and type IVb secretion systems. J. Biol. Chem. 287, 14157-14168. doi: 10.1074/jbc.M111.338731

Elias, W. P., Czeczulin, J. R., Henderson, I. R., Trabulsi, L. R., and Nataro, J. P. (1999). Organization of biogenesis genes for aggregative adherence fimbria II defines a virulence gene cluster in enteroaggregative Escherichia coli. J. Bacteriol. 181, 1779-85.

Franco, A. A., Hu, L., Grim, C. J., Gopinath, G., Sathyamoorthy, V., Jarvis, K. G., et al. (2011). Characterization of putative virulence genes on the related RepFIB plasmids harbored by Cronobacter spp. Appl. Environ. Microbiol. 77, 3255-3267. doi: 10.1128/AEM.03023-10 
Ghosal, D., Jeong, K. C., Chang, Y.-W., Gyore, J., Teng, L., Gardner, A., et al. (2019). Molecular architecture, polar targeting and biogenesis of the Legionella Dot/Icm T4SS. Nat. Microbiol. 4, 1173-1182. doi: 10.1038/s41564-019-0427-4

Gomis-Rüth, F. X., Solà, M., de la Cruz, F., and Coll, M. (2004). Coupling factors in macromolecular type-IV secretion machineries. Curr. Pharm. Des. 10, 1551-1565. doi: 10.2174/1381612043384817

Grohmann, E., Christie, P. J., Waksman, G., and Backert, S. (2018). Type IV secretion in Gram-negative and Gram-positive bacteria. Mol. Microbiol. 107, 455-471. doi: 10.1111/mmi.13896

Guglielmini, J., Quintais, L., Garcillán-Barcia, M. P., de la Cruz, F., and Rocha, E. P. C. (2011). The repertoire of ICE in prokaryotes underscores the unity, diversity, and ubiquity of conjugation. PLoS Genet. 7:e1002222. doi: 10.1371/journal.pgen.1002222

Guzmán-Herrador, D. L., and Llosa, M. (2019). The secret life of conjugative relaxases. Plasmid 104:102415. doi: 10.1016/j.plasmid.2019.102415

Halary, S., Leigh, J. W., Cheaib, B., Lopez, P., and Bapteste, E. (2010). Network analyses structure genetic diversity in independent genetic worlds. Proc. Natl. Acad. Sci. U.S.A. 107, 127-132. doi: 10.1073/pnas.0908978107

Ho, B. T., Basler, M., and Mekalanos, J. J. (2013). Type 6 secretion systemmediated immunity to type 4 secretion system-mediated gene transfer. Science 342, 250-253. doi: 10.1126/science. 1243745

Ishikawa, T., Rompikuntal, P. K., Lindmark, B., Milton, D. L., and Wai, S. N. (2009). Quorum sensing regulation of the two hcp alleles in Vibrio cholerae O1 strains. PLoS One 4, e6734. doi: 10.1371/journal.pone.0006734

Journet, L., and Cascales, E. (2016). The type VI secretion system in Escherichia coli and related species. EcoSal Plus 7, 1-20. doi: 10.1128/ecosalplus.ESP-0009-2015

Kostiuk, B., Unterweger, D., Provenzano, D., and Pukatzki, S. (2017). T6SS intraspecific competition orchestrates Vibrio cholerae genotypic diversity. Int. Microbiol. 20, 130-137. doi: 10.2436/20.1501.01.294

Kudryashev, M., Wang, R. Y.-R., Brackmann, M., Scherer, S., Maier, T., Baker, D., et al. (2015). Structure of the type VI secretion system contractile sheath. Cell 160, 952-962. doi: 10.1016/j.cell.2015.01.037

Leiman, P. G., Basler, M., Ramagopal, U. A., Bonanno, J. B., Sauder, J. M., Pukatzki, S., et al. (2009). Type VI secretion apparatus and phage tail-associated protein complexes share a common evolutionary origin. Proc. Natl. Acad. Sci. U.S.A. 106, 4154-4159. doi: 10.1073/pnas.0813360106

Lesic, B., Starkey, M., He, J., Hazan, R., and Rahme, L. G. (2009). Quorum sensing differentially regulates Pseudomonas aeruginosa type VI secretion locus I and homologous loci II and III, which are required for pathogenesis. Microbiology 155, 2845-2855. doi: 10.1099/mic.0.029082-0

Li, J., Yao, Y., Xu, H. H., Hao, L., Deng, Z., Rajakumar, K., et al. (2015). SecReT6: a web-based resource for type VI secretion systems found in bacteria. Environ. Microbiol. 17, 2196-2202. doi: 10.1111/1462-2920.12794

Li, Y. G., Hu, B., and Christie, P. J. (2019). Biological and structural diversity of type IV secretion systems. Microbiol. Spectr. 7, 1-15. doi: 10.1128/microbiolspec.PSIB-0012-2018

Liebl, D., Robert-Genthon, M., Job, V., Cogoni, V., and Attrée, I. (2019). Baseplate component TSSK and spatio-temporal assembly of T6SS in Pseudomonas aeruginosa. Front. Microbiol. 10:1615. doi: 10.3389/fmicb.2019. 01615

Lien, Y.-W., and Lai, E.-M. (2017). Type VI secretion effectors: methodologies and biology. Front. Cell. Infect. Microbiol. 7:254. doi: 10.3389/fcimb.2017.00254

Lossi, N. S., Dajani, R., Freemont, P., and Filloux, A. (2011). Structurefunction analysis of HsiF, a gp25-like component of the type VI secretion system, in Pseudomonas aeruginosa. Microbiology 157, 3292-3305. doi: 10.1099/mic.0.051987-0

Lossi, N. S., Manoli, E., Förster, A., Dajani, R., Pape, T., Freemont, P., et al. (2013). The HsiB1C1 (TssB-TssC) complex of the Pseudomonas aeruginosa type VI secretion system forms a bacteriophage tail sheathlike structure. J. Biol. Chem. 288, 7536-7548. doi: 10.1074/jbc.M112.439273

Majerczyk, C., Schneider, E., and Greenberg, E. P. (2016). Quorum sensing control of Type VI secretion factors restricts the proliferation of quorum-sensing mutants. Elife 5:e14712. doi: 10.7554/eLife.14712

Matthey, N., Stutzmann, S., Stoudmann, C., Guex, N., Iseli, C., and Blokesch, M. (2019). Neighbor predation linked to natural competence fosters the transfer of large genomic regions in Vibrio cholerae. Elife 8:e48212. doi: $10.7554 /$ eLife.48212
Morin, N., Santiago, A. E., Ernst, R. K., Guillot, S. J., and Nataro, J. P. (2013). Characterization of the AggR regulon in enteroaggregative Escherichia coli. Infect. Immun. 81, 122-132. doi: 10.1128/IAI.00676-12

Morin, N., Tirling, C., Ivison, S. M., Kaur, A. P., Nataro, J. P., and Steiner, T. S. (2010). Autoactivation of the AggR regulator of enteroaggregative Escherichia coli in vitro and in vivo. FEMS Immunol. Med. Microbiol. 58, 344-355. doi: 10.1111/j.1574-695X.2009.00645.x

Munson, G. P. (2013). Virulence regulons of enterotoxigenic Escherichia coli. Immunol. Res. 57, 229-236. doi: 10.1007/s12026-013-8453-4

Nataro, J. P., Yikang, D., Yingkang, D., and Walker, K. (1994). AggR, a transcriptional activator of aggregative adherence fimbria I expression in enteroaggregative Escherichia coli. J. Bacteriol. 176, 4691-4699. doi: 10.1128/jb.176.15.4691-4699.1994

Nazarov, S., Schneider, J. P., Brackmann, M., Goldie, K. N., Stahlberg, H., and Basler, M. (2018). Cryo-EM reconstruction of Type VI secretion system baseplate and sheath distal end. EMBO J. 37:e97103. doi: 10.15252/embj.201797103

Nguyen, V. S., Douzi, B., Durand, E., Roussel, A., Cascales, E., and Cambillau, C. (2018). Towards a complete structural deciphering of Type VI secretion system. Curr. Opin. Struct. Biol. 49, 77-84. doi: 10.1016/j.sbi.2018.01.007

Nishi, J., Sheikh, J., Mizuguchi, K., Luisi, B., Burland, V., Boutin, A., et al. (2003). The export of coat protein from enteroaggregative Escherichia coli by a specific ATP-binding cassette transporter system. J. Biol. Chem. 278, 45680-45689. doi: 10.1074/jbc.M306413200

Park, Y.-J., Lacourse, K. D., Cambillau, C., DiMaio, F., Mougous, J. D., and Veesler, D. (2018). Structure of the type VI secretion system TssK-TssF-TssG baseplate subcomplex revealed by cryo-electron microscopy. Nat. Commun. 9:5385. doi: 10.1038/s41467-018-07796-5

Peña, A., and Arechaga, I. (2013). Molecular motors in bacterial secretion. J. Mol. Microbiol. Biotechnol. 23, 357-369. doi: 10.1159/000351360

Pena, R. T., Blasco, L., Ambroa, A., González-Pedrajo, B., Fernández-García, L., López, M., et al. (2019). Relationship between quorum sensing and secretion systems. Front. Microbiol. 10:1100. doi: 10.3389/fmicb.2019.01100

Piel, D., Bruto, M., James, A., Labreuche, Y., Lambert, C., Janicot, A., et al. (2019). Selection of Vibrio crassostreae relies on a plasmid expressing a type 6 secretion system cytotoxic for host immune cells. Environ. Microbiol. doi: 10.1111/1462-2920.14776. [Epub ahead of print].

Pietrosiuk, A., Lenherr, E. D., Falk, S., Bönemann, G., Kopp, J., Zentgraf, H., et al. (2011). Molecular basis for the unique role of the AAA+ chaperone $\mathrm{ClpV}$ in type VI protein secretion. J. Biol. Chem. 286, 30010-30021. doi: $10.1074 /$ jbc.M111.253377

Planamente, S., Salih, O., Manoli, E., Albesa-Jové, D., Freemont, P. S., and Filloux, A. (2016). TssA forms a gp6-like ring attached to the type VI secretion sheath. EMBO J. 35, 1613-1627. doi: 10.15252/embj.201694024

Pukatzki, S., Ma, A. T., Revel, A. T., Sturtevant, D., and Mekalanos, J. J. (2007). Type VI secretion system translocates a phage tail spike-like protein into target cells where it cross-links actin. Proc. Natl. Acad. Sci. U.S.A. 104, 15508-15513. doi: 10.1073/pnas.0706532104

Rapisarda, C., Cherrak, Y., Kooger, R., Schmidt, V., Pellarin, R., Logger, L., et al. (2019). In situ and high-resolution cryo-EM structure of a bacterial type VI secretion system membrane complex. EMBO J. 38:e100886. doi: $10.15252 / \mathrm{embj} .2018100886$

Russell, A. B., Wexler, A. G., Harding, B. N., Whitney, J. C., Bohn, A. J., Goo, Y. A., et al. (2014). A type VI secretion-related pathway in Bacteroidetes mediates interbacterial antagonism. Cell Host Microbe 16, 227-236. doi: 10.1016/j.chom.2014.07.007

Salinero-Lanzarote, A., Pacheco-Moreno, A., Domingo-Serrano, L., Durán, D., Ormeño-Orrillo, E., Martínez-Romero, E., et al. (2019). The Type VI secretion system of Rhizobium etli Mim1 has a positive effect in symbiosis. FEMS Microbiol. Ecol. 95:fiz054. doi: 10.1093/femsec/fiz054

Santiago, A. E., Ruiz-Perez, F., Jo, N. Y., Vijayakumar, V., Gong, M. Q., and Nataro, J. P. (2014). A large family of antivirulence regulators modulates the effects of transcriptional activators in Gram-negative pathogenic bacteria. PLoS Pathog. 10:e1004153. doi: 10.1371/journal.ppat.1004153

Santiago, A. E., Yan, M. B., Tran, M., Wright, N., Luzader, D. H., Kendall, M. M., et al. (2016). A large family of anti-activators accompanying XylS/AraC family regulatory proteins. Mol. Microbiol. 101, 314-332. doi: 10.1111/mmi.13392 
Schneider, J. P., Nazarov, S., Adaixo, R., Liuzzo, M., Ringel, P. D., Stahlberg, H., et al. (2019). Diverse roles of TssA-like proteins in the assembly of bacterial type VI secretion systems. EMBO J. 38:e100825. doi: 10.15252/embj.20181 00825

Sheikh, J., Czeczulin, J. R., Harrington, S., Hicks, S., Henderson, I. R., Le Bouguénec, C., et al. (2002). A novel dispersin protein in enteroaggregative Escherichia coli. J. Clin. Invest. 110, 1329-1337. doi: 10.1172/JCI16172

Sheikh, J., Hicks, S., Dall'Agnol, M., Phillips, A. D., and Nataro, J. P. (2001). Roles for Fis and YafK in biofilm formation by enteroaggregative Escherichia coli. Mol. Microbiol. 41, 983-997. doi: 10.1046/j.1365-2958.2001.02512.x

Shneider, M. M., Buth, S. A., Ho, B. T., Basler, M., Mekalanos, J. J., and Leiman, P. G. (2013). PAAR-repeat proteins sharpen and diversify the type VI secretion system spike. Nature 500, 350-353. doi: 10.1038/nature12453

Shyntum, D. Y., Venter, S. N., Moleleki, L. N., Toth, I., and Coutinho, T. A. (2014). Comparative genomics of type VI secretion systems in strains of Pantoea ananatis from different environments. BMC Genomics 15:163. doi: 10.1186/1471-2164-15-163

Smillie, C., Garcillán-Barcia, M. P., Francia, M. V., Rocha, E. P. C., and de la Cruz, F. (2010). Mobility of plasmids. Microbiol. Mol. Biol. Rev. 74, 434-452. doi: 10.1128/MMBR.00020-10

Thomas, J., Watve, S. S., Ratcliff, W. C., and Hammer, B. K. (2017). Horizontal gene transfer of functional type VI killing genes by natural transformation. MBio 8::e00654-17. doi: 10.1128/mBio.00654-17

Uchida, K., Leiman, P. G., Arisaka, F., and Kanamaru, S. (2014). Structure and properties of the C-terminal $\beta$-helical domain of VgrG protein from Escherichia coli O157. J. Biochem. 155, 173-182. doi: 10.1093/jb/mvt109

Veening, J.-W., and Blokesch, M. (2017). Interbacterial predation as a strategy for DNA acquisition in naturally competent bacteria. Nat. Rev. Microbiol. 15, 621-629. doi: 10.1038/nrmicro.2017.66

Veesler, D., and Cambillau, C. (2011). A common evolutionary origin for tailedbacteriophage functional modules and bacterial machineries. Microbiol. Mol. Biol. Rev. 75, 423-33. doi: 10.1128/MMBR.00014-11

Verster, A. J., Ross, B. D., Radey, M. C., Bao, Y., Goodman, A. L., Mougous, J. D., et al. (2017). The landscape of type VI secretion across human gut microbiomes reveals its role in community composition. Cell Host Microbe 22, 411-9.e4. doi: 10.1016/j.chom.2017.08.010

Wang, J., Brackmann, M., Castaño-Díez, D., Kudryashev, M., Goldie, K. N., Maier, T., et al. (2017). Cryo-EM structure of the extended type VI secretion system sheath-tube complex. Nat. Microbiol. 2, 1507-1512. doi: 10.1038/s41564-017-0020-7
Wang, J., Brodmann, M., and Basler, M. (2019). Assembly and subcellular localization of bacterial type VI secretion systems. Annu. Rev. Microbiol. 73, 621-638. doi: 10.1146/annurev-micro-020518-115420

Weber, B. S., Ly, P. M., Irwin, J. N., Pukatzki, S., and Feldman, M. F. (2015), A multidrug resistance plasmid contains the molecular switch for type VI secretion in Acinetobacter baumannii. Proc. Natl. Acad. Sci. U.S.A. 112, 9442-9447. doi: 10.1073/pnas.1502966112

Weber, B. S., Miyata, S. T., Iwashkiw, J. A., Mortensen, B. L., Skaar, E. P., Pukatzki, S., et al. (2013). Genomic and functional analysis of the type VI secretion system in Acinetobacter. PLoS ONE 8:e55142. doi: 10.1371/journal.pone.0055142

Yang, Z., Zhou, X., Ma, Y., Zhou, M., Waldor, M. K., Zhang, Y., et al. (2018). Serine/threonine kinase PpkA coordinates the interplay between T6SS2 activation and quorum sensing in the marine pathogen Vibrio alginolyticus. Environ. Microbiol. 20, 903-919. doi: 10.1111/1462-2920.14039

Yasir, M., Icke, C., Abdelwahab, R., Haycocks, J. R., Godfrey, R. E., Sazinas, P., et al. (2019). Organization and architecture of AggR-dependent promoters from enteroaggregative Escherichia coli. Mol. Microbiol. 111, 534-551. doi: $10.1111 / \mathrm{mmi} .14172$

Zechner, E. L., Moncalián, G., and de la Cruz, F. (2017). Relaxases and plasmid transfer in gram-negative bacteria. Curr. Top. Microbiol. Immunol. 413, 93-113. doi: 10.1007/978-3-319-75241-9_4

Zhang, L., Xu, J., Xu, J., Zhang, H., He, L., and Feng, J. (2014). TssB is essential for virulence and required for type VI secretion system in Ralstonia solanacearum. Microb. Pathog. 74, 1-7. doi: 10.1016/j.micpath.2014.06.006

Zheng, J., Shin, O. S., Cameron, D. E., and Mekalanos, J. J. (2010). Quorum sensing and a global regulator TsrA control expression of type VI secretion and virulence in Vibrio cholerae. Proc. Natl. Acad. Sci. U.S.A. 107, 21128-21133. doi: 10.1073/pnas.1014998107

Conflict of Interest: The authors declare that the research was conducted in the absence of any commercial or financial relationships that could be construed as a potential conflict of interest.

Copyright $\odot 2019$ Peñil-Celis and Garcillán-Barcia. This is an open-access article distributed under the terms of the Creative Commons Attribution License (CC BY). The use, distribution or reproduction in other forums is permitted, provided the original author(s) and the copyright owner(s) are credited and that the original publication in this journal is cited, in accordance with accepted academic practice. No use, distribution or reproduction is permitted which does not comply with these terms. 